\title{
Optimizing the Power Budget of Hovering AUVs
}

\author{
Nuno A. Cruz \\ Faculty of Engineering, University of Porto and INESC TEC \\ Rua Dr. Roberto Frias, 4200-465 Porto, Portugal \\ nacruz@fe.up.pt
}

\begin{abstract}
The maximum mission duration and range of an Autonomous Underwater Vehicle are governed by the amount of energy carried on board and the way it is spent during the mission. While an increase in battery capacity and a decrease in electronics demand yield a direct increase in vehicle range, the impact of velocity variation is not so obvious. With slower velocities, most of the energy will be spent in electronics, not in motion, while for faster velocities a lot of energy will be needed to balance drag. Flying-type AUVs have a minimum velocity for the control surfaces to be effective, reducing the range of values for optimization. Hovering type AUVs, on the other hand, are typically slower moving platforms, able to travel at arbitrarily slow velocities. This paper addresses the analysis of the power consumption of hovering type AUVs, providing guidelines and analytical expressions to compute the optimal velocity when the vehicle travels in a single direction, and also when the trajectory is a combination of horizontal and vertical motion.
\end{abstract}

\section{INTRODUCTION}

The last decades have witnessed the proliferation of $\mathrm{Au}$ tonomous Underwater Vehicles (AUVs) in multiple scenarios of underwater exploration. Most earlier vehicles were of the flying type, i.e vehicles designed to travel long distances and sample the largest possible volumes of water. Hovering type AUVs, on the other hand, are typically slower moving platforms, able to travel at arbitrarily slow velocities, and therefore able to approach the sea bottom for close-range inspections.

Given the difficulty to harvest energy from the environment, virtually all AUVs need to transport the energy needed for each mission, typically using some form of rechargeable batteries. Therefore, it is no surprise that the analysis of the power consumption, and it's impact in mission duration, has been a topic of study ever since the earlier deployments. In most of these studies, vehicles would be programmed to follow a predetermined path at a constant optimal velocity, transporting a set of sensors that would require a relatively fixed amount of power. Such an optimal velocity could be estimated from the characteristics of the propulsion system and from all other on board components that would require energy from the same source. In these cases, the main objective was typically to increase range.

Hovering AUVs have the capability of holding position in the middle of the water column, or approach the sea bottom for close range inspection or other local survey, adding to the standard capability of traveling long distances. In fact, hovering type AUVs have significantly enlarged the scope of typical mission profiles, and many missions are now measured in terms of hours of operation (for example, taking high resolution videos of the sea bottom), instead of kilometers. Moreover, current mission profiles frequently include the ability to switch sensors on and off, that make it more complex to estimate general energy usage and optimize mission profiles. In these cases, the traditional concept of autonomy as spatial range can be misleading as the vehicle can spend all its energy in a very limited area.

This paper addresses the analysis of the power consumption of hovering type AUVs. AUV missions are usually specified as a sequence of maneuvers. When a maneuver is fully determined in terms of time, range and velocity, the total amount of energy required for that maneuver can be computed, and subtracted from the total amount available. The optimization process is useful for the case when those parameters are not set and the objective is to extend the duration/range of the mission or maneuver.

The paper illustrates the above mentioned optimization with data from a hovering-type AUV that has been developed at INESC TEC, the MARES AUV [1]. This analysis is provided with simple steps that can easily be adapted to optimize the mission range of other hovering AUVs.

The paper is organized as follows. Section II provides an overview of other analysis of power budget for underwater missions with AUVs. Section III summarizes the main characteristics of the class of vehicles used for the case study. Section IV describes the optimization process when the vehicle travels in a single direction, either in the horizontal plane, or purely vertically, while section $\mathrm{V}$ takes into account the combination of vertical and horizontal motion. Lastly, in Section VI we present some concluding remarks and directions for future work.

\section{RELATED WORK}

On board energy is one of the most valuable assets of an AUV, and the ability to spend it wisely affects its operational effectiveness. The most common AUV propulsion system is based on thrusters, either vectored or with the aid of fins to control direction and pitch angle. This results in the so-called "flying-type"' AUV, a design that requires a minimum velocity for the control surfaces to be effective. During an AUV mission, many electronic systems need to be continuously working (CPU, navigation system, payload sensors, etc.), therefore the maximum range is dictated by a combination of their power consumption, together with power required for motion. This, in turn is mostly spent to overcome 
vehicle drag, which grows with the square of the velocity. In [2], Willcox et al. have focused on the power demand of these flying type AUVs, computing the optimal velocity that minimizes the energy consumption per unit distance traveled, therefore resulting in the longest range of operation for a given power profile.

The trend to extend the duration of AUV missions has been taking advantage of the increased energy density of batteries, together with a more efficient use. For example, the Tethys AUV, developed at the Monterey Bay Aquarium Research Institute, is a $105 \mathrm{~kg}$ propeller-driven vehicle that integrates a variable buoyancy system in a very efficient energy management system. Hobson et al., in [3], report a three week deployment covering $1800 \mathrm{~km}$ at a speed of $1 \mathrm{~m} / \mathrm{s}$, with a suite of payload sensors averaging 5 watt of power consumption. The authors analyse the power consumption in different regimes of operation to compute the optimal velocity for increased range, achieving an estimated maximum of 3 thousand kilometers, during months of operation.

\section{Modular Hovering AUVs}

The concept of modular robotics has been proposed as a way to rapidly assemble and reconfigure systems for specific purposes. At INESC TEC, this concept has been adopted for the development of small size AUVs based on modular building blocks [4]. This approach relies on modularity both in terms of hardware construction, but also in terms of electronics, software and control. The design of the hull sections is based on cylindrical shapes with matching edges and constant cross sections, with $20 \mathrm{~cm}$ of outer diameter.

Using these modular building blocks, the first version of the MARES portable AUV was built in 2007 [1]. MARES is a shallow water $(100 \mathrm{~m})$ hovering AUV that has been continuously updated and used in the field in many different configurations. On a basic configuration, the vehicle has little over $1.7 \mathrm{~m}$ of length and $35 \mathrm{~kg}$. The vehicle has a total of $600 \mathrm{Wh}$ of energy and can be configured with an assortment of sensors, with two horizontal thrusters to control surge and yaw, and two through-hull vertical thrusters to control heave and pitch, providing hovering capability.

With a different set of system components, the TriMARES was developed in 2011 [5]. TriMARES is a $75 \mathrm{~kg}$, 3-body hybrid AUV/ROV system, which was developed and delivered to a Brazilian consortium in little over 6 months. The vehicle has a total of $800 \mathrm{Wh}$ of energy, and uses 7 independent thrusters: 4 to control surge and yaw, 2 vertical for pitch and heave, and a lateral thruster for sway. More recently, the DART AUV (for Deep water Autonomous Robotic Traveler) has been assembled, with the replacement of the main pressure housing of MARES with a 4,000 $\mathrm{m}$ rated enclosure [6]. The vehicle has the same thruster configuration as the MARES AUV, therefore also being able to hover in the water column. Figure 1 shows pictures of these vehicles.

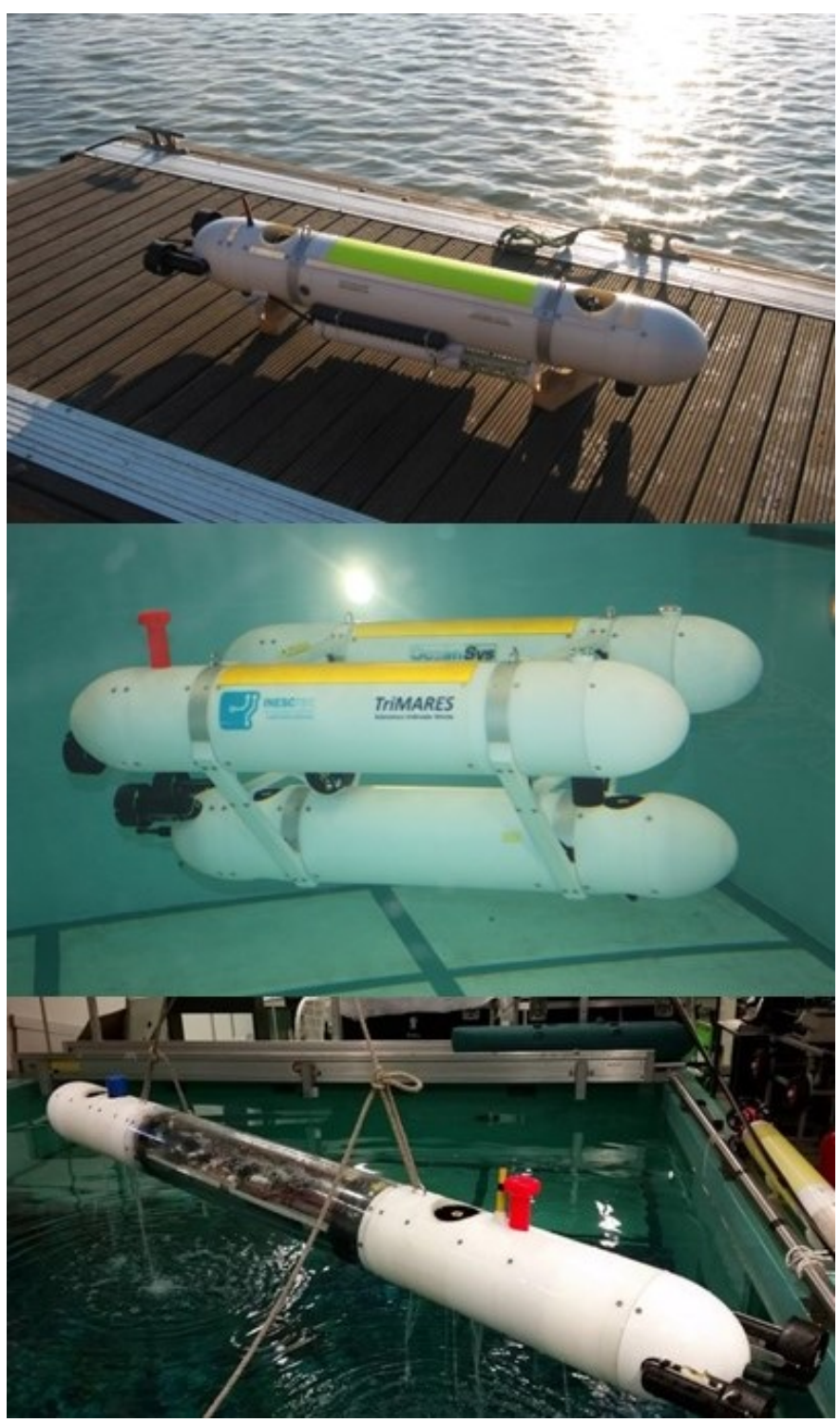

Fig. 1. MARES, TriMARES and DART (top to bottom), three example of hovering AUVs assembled from a set of modular building blocks.

\section{Velocity Optimization IN A SiNGLE DiRECTION}

In a purely horizontal motion, the horizontal thrusters have to provide enough power to balance the steady state drag force acting on the submerged body. This force is proportional to the square of the horizontal velocity (or surge), $u$, and it is given by:

$$
F_{d}=\frac{1}{2} \rho C_{d} A u^{2}=K_{d u} u^{2}
$$

where $\rho$ is the density of the water (about $1,000 \mathrm{~kg} / \mathrm{m}^{3}$ ), $C_{d}$ is the drag coefficient (depending on the shape of the AUV), and $A$ is the projected area. For simplicity, $K_{d u}$ aggregates all the constants related to surge motion.

If we assume that the thrusters have an overall efficiency of $\eta$, then the energy required to move the AUV over a horizontal 
TABLE I

DYNAMIC PARAMETERS OF THE MARES AUV

\begin{tabular}{|c|c|c|}
\hline Symbol & Value & Description \\
\hline$E_{t}$ & $2.16 \times 10^{6} \mathrm{~J}$ & Available energy \\
$P_{h}$ & $15 \mathrm{~W}$ & Hotel load \\
$\eta$ & 0.15 & Propulsion efficiency \\
$K_{d u}$ & $4 \mathrm{~kg} \mathrm{~m}^{-1}$ & Drag constant (horizontal) \\
$K_{d w}$ & $200 \mathrm{~kg} \mathrm{~m}^{-1}$ & Drag constant (vertical) \\
$d$ & $4.5 \times 10^{-3} \mathrm{~m}$ & $\mathrm{C}_{B} / \mathrm{C}_{G}$ separation \\
$b$ & $0.85 \mathrm{~m}$ & Vertical thrusters separation \\
\hline
\end{tabular}

distance $R_{h}$ is given by:

$$
E_{m}=\frac{F_{d}}{\eta} R_{h}=\frac{K_{d u}}{\eta} u^{2} R_{h}
$$

Consider that all the other on-board subsystems need an average power (typically called hotel load) of $P_{h}$. In this case, the total instantaneous power used by the vehicle, $P_{t}$, is the sum of this fixed part, with the power required for motion, $P_{m}$ :

$$
P_{t}=P_{h}+P_{m}=P_{h}+\frac{K_{d u}}{\eta} u^{3}
$$

The energy draw from the batteries, when the AUV travels a horizontal distance $R_{h}$ with average surge velocity $u$, can be calculated as:

$$
E_{t}=P_{h} \frac{R_{h}}{u}+\frac{K_{d u}}{\eta} u^{2} R_{h}=\left(\frac{P_{h}}{u}+\frac{K_{d u}}{\eta} u^{2}\right) R_{h}
$$

This expression can be rewritten so that we can estimate the maximum horizontal range of the AUV, depending on the surge velocity, $u$ :

$$
R_{h}=\frac{E_{t}}{\frac{P_{h}}{u}+\frac{K_{d u}}{\eta} u^{2}}
$$

The previous analysis of equations $1-5$ can be similarly performed for the case of the vehicle performing a purely vertical motion, i.e. with zero surge and only actuating the vertical thrusters to provide heave. This is particularly relevant when the vehicle is required to perform yo-yo's.

In order to estimate the quantities described in these equations for the case of MARES, we can use the set of parameters summarized in table I. The hydrodynamic parameters were calculated following the approach in [7] and using some typical values found in [8]. Figures 2 and 3, show how the variation of surge and heave affect the horizontal and vertical range, respectively.

Although the graphical analysis of figures 2 and 3 serves to identify the peaks and the variation around them, it is possible to derive an analytical solution to the optimal velocity, that is, the velocity that yields a maximum horizontal distance traveled, by taking the derivative of equation 5 with respect to the surge velocity, $u$ :

$$
\frac{d R_{h}}{d u_{o p t}}=0 \Rightarrow u_{o p t}=\left(\frac{P_{h} \eta}{2 K_{d u}}\right)^{\frac{1}{3}}
$$
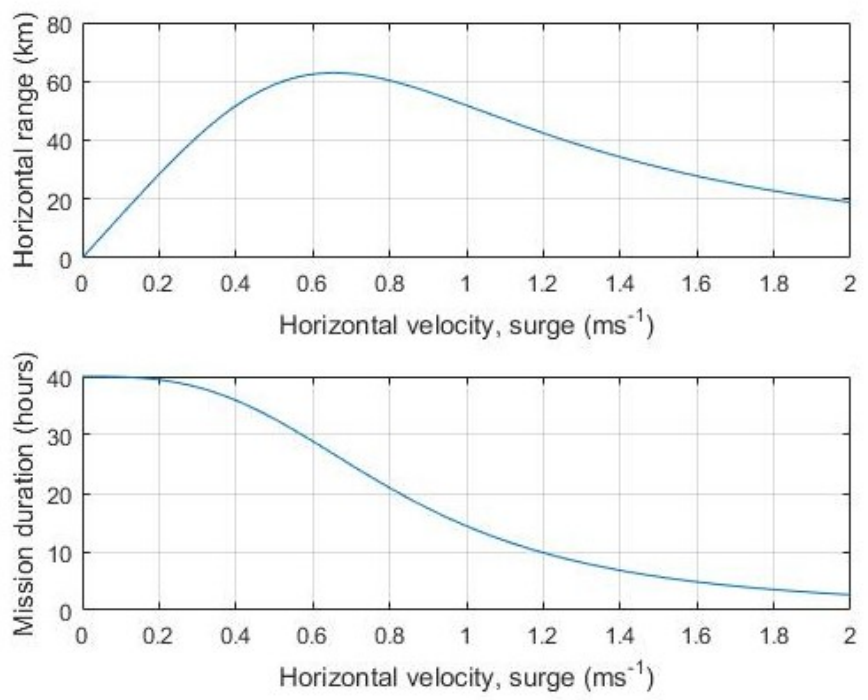

Fig. 2. Autonomy of the MARES AUV as a function of horizontal velocity.
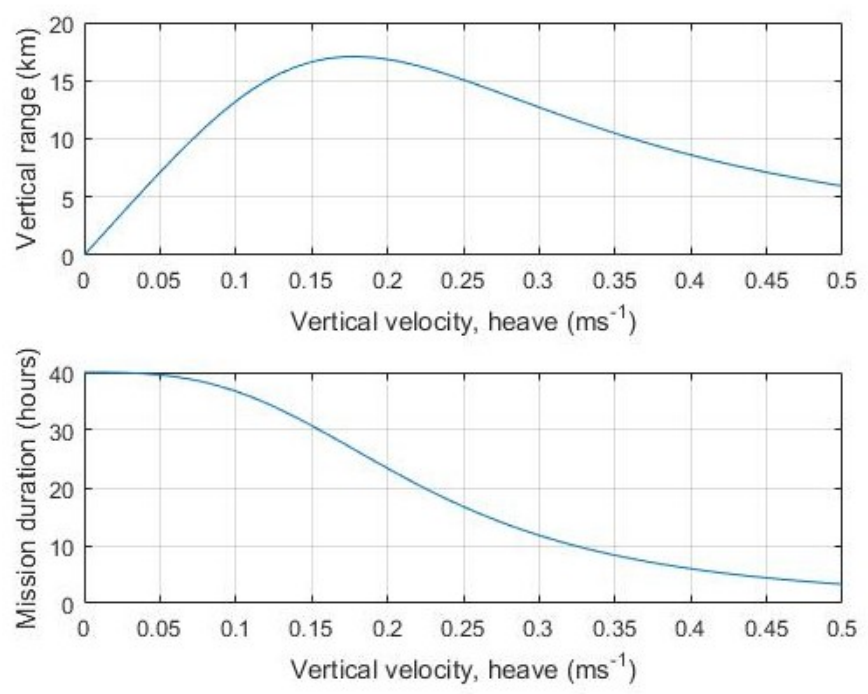

Fig. 3. Autonomy of the MARES AUV as a function of vertical velocity.

Note that the optimal velocity is independent of the amount of energy available on board. Furthermore, at this velocity, the total instantaneous power used by the vehicle (see eq. 3) is $3 / 2 P_{h}$, resulting in a maximum operating time of:

$$
t_{\max }=\frac{E_{t}}{\frac{3}{2} P_{h}}
$$

and a maximum range of:

$$
R_{h_{\max }}=\frac{E_{t}\left(\frac{\eta}{2 K_{d u}}\right)^{\frac{1}{3}}}{\frac{2}{3} P_{h}^{\frac{2}{3}}}
$$

Using the parameters of table $I$ in equation 6 results in an optimal velocity of $u_{o p t}=0.66 \mathrm{~ms}^{-1}$ for the MARES 

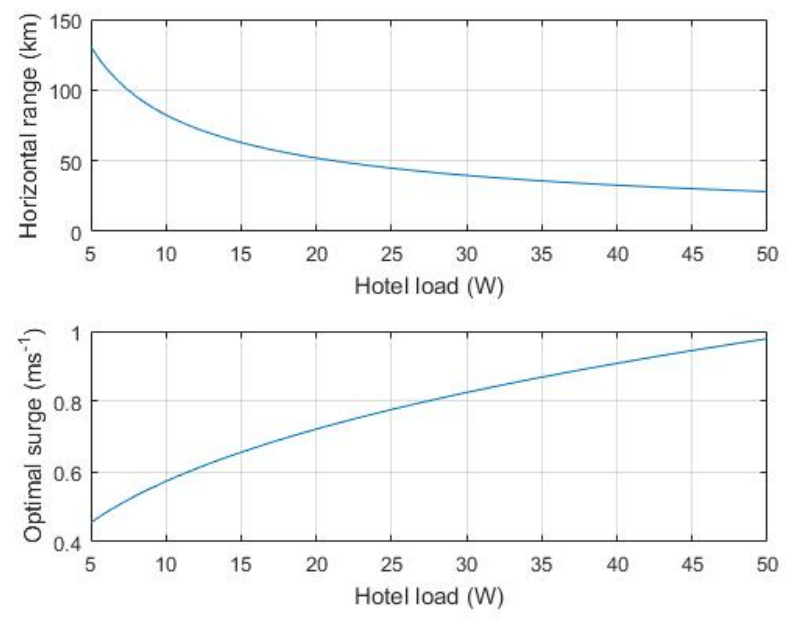

Fig. 4. Influence of hotel load in mission range and optimal surge velocity.

AUV, corresponding to a maximum horizontal range to be $R_{h_{\max }}=63 \mathrm{~km}$, and a mission lasting over 26 hours. Similarly, doing the same exercise for the vertical motion, yields an optimal heave velocity of $w_{\text {opt }}=0.18 \mathrm{~ms}^{-1}$, corresponding to a maximum vertical range $R_{v_{\max }}=17 \mathrm{~km}$, or 85 full cycles from the surface to the maximum depth of $100 \mathrm{~m}$.

These values are estimates that may be affected by practical limitations. For example, even in purely horizontal missions, the vertical thrusters need to be used to provide a steady state downward force to compensate for a slight vehicle buoyancy usually adopted for safety reasons. Naturally, they also need to be used by the on-board controllers to compensate for any disturbances in pitch and depth. All this actuation requires a steady state power that will add to the hotel load, $P_{h}$, and will degrade the estimation of maximum range. The same applies if the AUV operates with payload sensors that demand a continuous supply of power. Figure 4 shows the influence of the total hotel load in the maximum mission range and optimal surge velocity, from a reduction to $5 \mathrm{~W}$ to an increase to a total of $50 \mathrm{~W}$.

\section{OPTIMIZATION IN COUPLED HORIZONTAL AND VERTICAL MOTION}

Coupling horizontal and vertical motion is very typical in oceanographic missions, with data being gathered in yoyo patterns to capture 3D snapshots of scalar fields. Since most of oceanographic data shows greater variations in the vertical plane as compared to the horizontal plane, it is usually necessary to travel more in the horizontal plane than in the vertical, therefore the horizontal component of the velocity is typically higher than the vertical.

MARES has two independent vertical thrusters, located away from the center of mass (see top of fig. 1), that provide the capability to control both pitch and vertical velocity. With this setting, there are two possibilities of producing a trajectory with inclination $\alpha_{t}$. The first option is to assume a

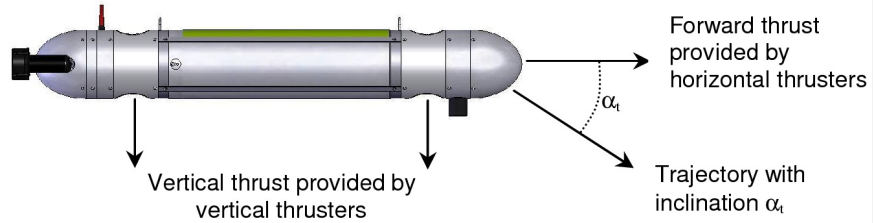

Fig. 5. Coupled horizontal and vertical motion with zero pitch angle.

zero pitch angle and provide both horizontal velocity (surge) and common-mode vertical velocity (heave) as represented in figure 5. This mode of operation may be very useful whenever it is important to keep track of the bottom, for example during sonar or video mapping in constant altitude above the sea floor (in bottom following). In this case, the steady attitude in pitch allows for the acquisition of very smooth data.

In order to evaluate the mission endurance in this configuration, we assume, for simplification, that there are two separate drag forces that can be considered independently: one horizontal, similar to the case of pure horizontal motion mentioned above, and an additional vertical component, that has to be balanced by the vertical thrusters.

The drag coefficient for the vertical motion of an AUV, $K_{d w}$, is much higher than the above $K_{d u}$ due to the significant projected area and less hydrodynamic shape (in the case of MARES, $K_{d w} \approx 200$, as indicated in table I). If we assume that the vehicle will be moving at a constant vertical velocity, $w$, then the vertical thrusters will have to balance a constant drag force of $F_{d w}=K_{d w} w^{2}$.

To ensure an inclination $\alpha_{t}$, the vertical component of the velocity has to be $w=u \tan \left(\alpha_{t}\right)$ and the distance traveled in the vertical plane is given by $R_{v}=R_{h} \tan \left(\alpha_{t}\right)$, where $R_{h}$ is the horizontal distance. The total energy required for motion is now given by the combination of the fractions for horizontal and vertical motion, given by

$$
\begin{array}{r}
E_{m}=\frac{K_{d u}}{\eta} u^{2} R_{h}+\frac{K_{d w}}{\eta} w^{2} R_{h} \tan \left(\alpha_{t}\right)= \\
=\frac{K_{d u}+K_{d w} \tan ^{3}\left(\alpha_{t}\right)}{\eta} u^{2} R_{h}
\end{array}
$$

Comparing with equation 2 above, the result of including a vertical motion can be seen as the same of increasing the drag coefficient. The new equivalent drag is now $K_{d}=K_{d u}+K_{d w} \tan ^{3}\left(\alpha_{t}\right)$. Figure 6 shows the variation in horizontal range, when there is a vertical component of the velocity, for values of trajectory inclination $\alpha_{t}$ between $0^{\circ}$ and $30^{\circ}$. We assume for simplicity that the vehicle is neutrally buoyant, which means that all vertical thrust is used to change the inclination of the trajectory. Note that the AUV cannot travel simultaneously with large velocities and steep trajectory inclination, due to the saturation in the vertical component of the velocity. This is the reason for the incomplete lines in the graphs of figure 6.

The analysis of the plots of figure 6 shows that for relatively small angles of the trajectory $\left(\alpha_{t}<15^{\circ}\right)$, the deterioration of 


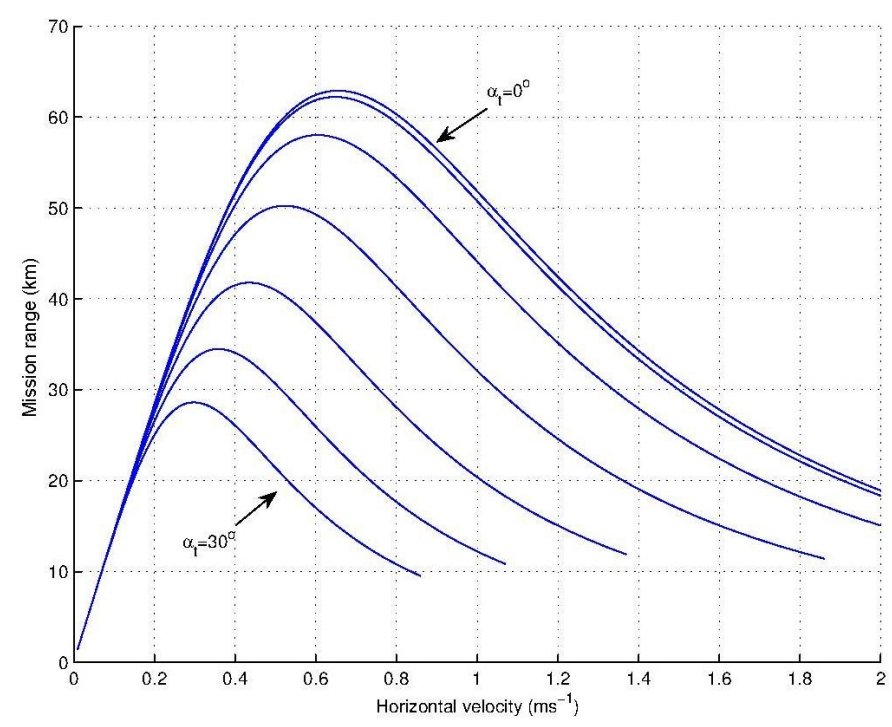

Fig. 6. Horizontal range of the MARES AUV as a function of the horizontal velocity, assuming zero pitch and trajectory inclination $\alpha_{t}$ between $0^{\circ}$ and $30^{\circ}$, in $5^{\circ}$ intervals.

the maximum range is not very high $(<20 \%)$, but as the angle of the trajectory increases, this maximum range occurs for smaller horizontal velocities. For example, the plots indicate that the vehicle can perform vertical yo-yo's with an inclination of $15^{\circ}$ during approximately $50 \mathrm{~km}$, at a horizontal velocity of $0.5 \mathrm{~ms}^{-1}$. If the angle increases to $30^{\circ}$, then the range is less than $30 \mathrm{~km}$ and has to be done at only $0.3 \mathrm{~ms}^{-1}$. This deterioration is natural since the trajectory is dictated by the ratio between surge and heave, and the power required to provide motion is much higher in the vertical plane than in the horizontal, due to drag.

In the above analysis we assumed that both vertical thrusters contributed to the vertical component of the velocity, i.e. the AUV had only common mode actuation and the resulting vehicle pitch was zero, even though the longitudinal trajectory had an inclination $\alpha_{t}$. A second option in terms of vertical motion is to use a differential mode of the vertical thrusters to provide a torque and therefore achieving a steady state pitch angle, $\alpha_{t}$. The horizontal thrusters will then push the AUV forward (in the longitudinal direction), resulting in the same spatial trajectory as above, but this time with a pitch angle different from zero, aligned with the trajectory (figure 7).

In this mode of operation, the horizontal velocity, $u_{h}$, is the projection of the longitudinal velocity (surge), $u$, into the horizontal plane:

$$
u_{h}=u \cos \left(\alpha_{t}\right)
$$

As far as energy is concerned, we still need to overcome the drag associated with surge, but now the energy required to maintain a steady state pitch angle has also to be accounted for. A neutrally buoyant underwater body with weight $W=m g$ and a separation of $d$ between center of buoyancy and center of mass, will have a righting moment if pitching at an angle $\alpha_{t}$,

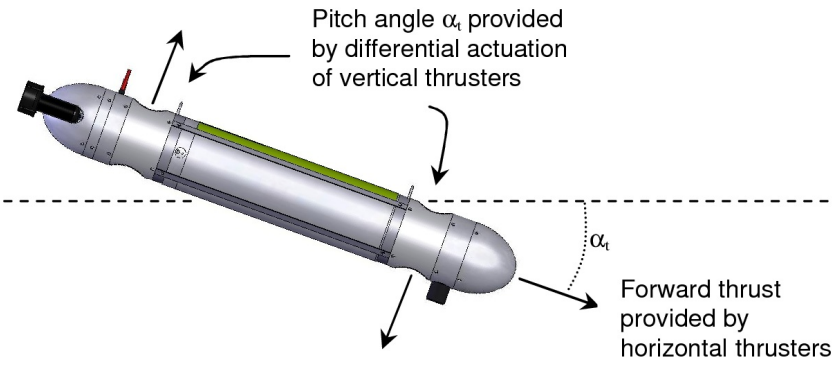

Fig. 7. Coupled horizontal and vertical motion controlling the pitch angle aligned with the intended trajectory.

given by $M=W d \sin \left(\alpha_{t}\right)$. Therefore, to maintain this steady state pitch angle $\alpha_{t}$, the vertical thrusters have to balance this moment, and so

$$
F_{T} b=M=W d \sin \left(\alpha_{t}\right)
$$

where $F_{T}$ is the force of each vertical thruster and $b$ is the separation between thrusters. Note that we assume for simplicity that the vertical thrusters are symmetrical with respect to the center of mass, and we also assume that thrusters behave equally working in forward or reverse modes, which is not entirely accurate. However, some of these asymmetries can be compensated in software, by using proper calibration curves, and this analysis can be easily expanded to accommodate the exact characteristics of the propulsion system.

In MARES, the electrical power required to provide a force $F_{T}$ in each thruster can be approximated by

$$
P_{T} \approx k_{T} F_{T}
$$

with $k_{T}$ estimated from experimental results to be $k_{T} \approx 5$.

So, we can derive the power required to achieve a steady state pitch angle of $\alpha_{t}$ as

$$
P_{T}=2 k_{T} \frac{W d}{b} \sin \left(\alpha_{t}\right)
$$

If we wish to analyse the energy expenditure with respect to horizontal range, then we have to consider all the energy spent on a mission with average horizontal velocity $u_{h}$ :

$$
E_{t}=2 k_{T} \frac{W d}{b} \sin \left(\alpha_{t}\right) \frac{R_{h}}{u_{h}}+P_{h} \frac{R_{h}}{u_{h}}+\frac{K_{d u}}{\eta \cos ^{2}\left(\alpha_{t}\right)} u_{h}^{2} R_{h}
$$

or

$$
E_{t}=\left(\frac{2 k_{T} \frac{W d}{b} \sin \left(\alpha_{t}\right)+P_{h}}{u_{h}}+\frac{K_{d u}}{\eta \cos ^{2}\left(\alpha_{t}\right)} u_{h}^{2}\right) R_{h}
$$

This means that the horizontal range can be directly estimated as:

$$
R_{h}=\frac{E_{t}}{\frac{2 k_{T} \frac{W d}{b} \sin \left(\alpha_{t}\right)+P_{h}}{u_{h}}+\frac{K_{d u}}{\eta \cos ^{2}\left(\alpha_{t}\right)} u_{h}^{2}}
$$

Comparing equation 17 with 5 , we can verify that the effect of a steady state pitch angle is the same as increasing the vehicle hotel load, and that the horizontal velocity is now a projection of the velocity vector into the horizontal plane. 


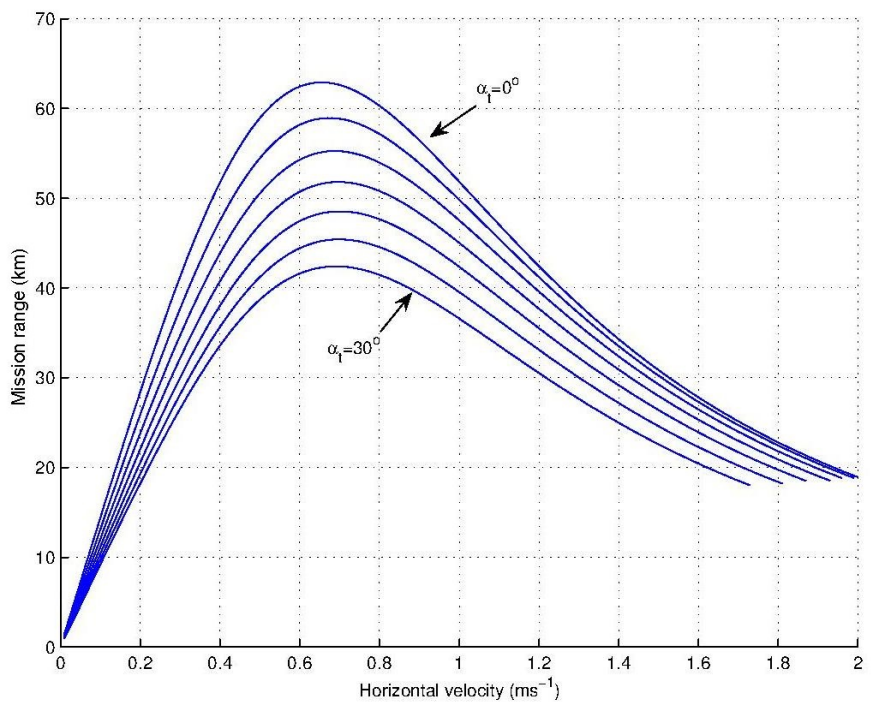

Fig. 8. Horizontal range of the MARES AUV as a function of the horizontal velocity, assuming that the pitch angle is aligned with the trajectory inclination $\alpha_{t}$, between $0^{\circ}$ and $30^{\circ}$, in $5^{\circ}$ intervals.

Figure 8 shows the variation in horizontal range, for values of AUV/trajectory inclination $\alpha_{t}$ between $0^{\circ}$ and $30^{\circ}$. Note that MARES longitudinal velocity is limited by the power in the stern thrusters to approximately $2 \mathrm{~ms}^{-1}$ and, therefore, the increase in trajectory inclination reduces the maximum horizontal component of the velocity, $u_{h}$. Once again, this shows up as incomplete lines in the graphs of figure 8 . The separation of the plots in figure 8 is dictated by the amount of power required to change the pitch, which is directly related to the righting moment and inversely related to the separation between the vertical thrusters.

From the comparison between figures 8 and 6 it is possible to observe that the best mechanism to provide a spatial inclination in the trajectory (for example, to follow a yo-yo pattern) depends on the angle of inclination that is required and also on the velocity of the survey. If the only requirement is the inclination angle of the trajectory, then the AUV is more efficient with a zero-pitch for small trajectory inclinations. If there is also any requirement in terms of minimum velocity to reduce survey time, then the AUV should adopt the same pitch as the desired trajectory for higher velocities. Note that MARES has a relatively small separation between the center of buoyancy and the center of mass (only 4.5 milimeters) and a large separation between the vertical thrusters. This results in a very small power required for the vertical thrusters to change pitch angle. If this separation increases (which is good for motion stability), then the effort required to overcome the righting moment will also increase and the most efficient way to descend will be with zero pitch. Naturally that the above figures and conclusions should be used as tools to design AUV missions, but they may change if the vehicle configuration is altered, for example with the addition of new sensors or actuators.

\section{CONCLusion And Future Work}

Given the limited amount of energy stored in a typical AUV, it is very important to use it efficiently. Most of the power required by an AUV is usually spent in propulsion, and so battery endurance greatly depends on vehicle velocity.

This paper presents an analytical computation of the optimal velocity of an AUV, taking into account the overall power budget of vehicle subsystems. This optimal value minimizes the energy consumption per unit distance traveled, therefore maximizing mission range. If needed, this computation may be easily performed in real time due to the analytical formulation, to adjust for dynamic variation of the relevant parameters.

As a case study, the paper provides data regarding the MARES AUV in a standard configuration, but it can easily be adapted to other vehicles or configurations, for which the profile of power consumption is known. In fact, we are using this approach to design parts of AUV missions where the velocity is not critical and can be specified to increase range. This includes mapping and other cases where we want to increase coverage for each charge of the batteries.

In the near future, we plan to integrate these calculations in the mission planning software, not only to automatically set the maneuver velocity, but also to do it in real time, based on exact measurements on power consumption and energy for the thrusters. Note, for example, that operations in estuaries result in buoyancy variations that are extremely hard to predict, and these result in variations in steady state consumption to ensure depth control.

\section{ACKNOWLEDGMENT}

This work was developed within the scope of INTENDU, a research project funded by the ERA-NET Cofund MarTERA under grant MARTERA/0001/2017, managed by the Portuguese funding agency FCT - Fundação para a Ciência e a Tecnologia (Foundation for Science and Technology).

\section{REFERENCES}

[1] N. A. Cruz and A. C. Matos, "The MARES AUV, a modular autonomous robot for environment sampling," in Proc. MTS/IEEE Int. Conf. Oceans 2008, Quebec, Canada, Sept. 2008.

[2] J. S. Willcox, J. G. Bellingham, Y. Zhang, and A. B. Baggeroer, "Performance metrics for oceanographic surveys with autonomous underwater vehicles," IEEE J. Oceanic. Eng., vol. 26, no. 4, pp. 711-725, Oct. 2001.

[3] B. W. Hobson, J. G. Bellingham, B. Kieft, R. McEwen, M. Godin, and Y. Zhang, "Tethys-class long range AUVs - extending the endurance of propeller-driven cruising AUVs from days to weeks," in Proc. IEEE/OES Conf. Autonomous Underwater Vehicles AUV 2012, Southampton, UK, Sept. 2012

[4] N. A. Cruz, A. C. Matos, and B. M. Ferreira, "Modular building blocks for the development of AUVs - from MARES to TriMARES," in Proc. Int. Symp. Underwater Tech. UT'13, Tokyo, Japan, Mar. 2013.

[5] N. A. Cruz, A. C. Matos, R. M. Almeida, B. M. Ferreira, and N. Abreu, "TriMARES - a hybrid AUV/ROV for dam inspection," in Proc. MTS/IEEE Int. Conf. Oceans'11, Kona, HI, USA, Sep. 2011.

[6] N. A. Cruz, A. C. Matos, R. M. Almeida, and B. M. Ferreira, "DART - a portable deep water hovering AUV," in Proc. MTS/IEEE Int. Conf. Oceans'17, Anchorage, AK, USA, Sept. 2017.

[7] T. Prestero, "Verification of a six-degree of freedom simulation model for the REMUS autonomous underwater vehicle," Master's thesis, Massachusetts Institute of Technology and Woods Hole Oceanographic Institution, Sep. 2001.

[8] S. F. Hoerner, Fluid-Dynamic Drag. Published by author, 1965. 